\title{
STABLE STATIONARY STATES OF NON-LOCAL INTERACTION EQUATIONS
}

\author{
KLEMENS FELLNER AND GAËL RAOUL
}

\begin{abstract}
In this article, we are interested in the large-time behaviour of a solution to a non-local interaction equation, where a density of particles/individuals evolves subject to an interaction potential and an external potential. It is known that for regular interaction potentials, stable stationary states of this equations are generically finite sums of Dirac masses.

For a finite sum of Dirac masses, we give i) a condition to be a stationary state, ii) two necessary conditions of linear stability w.r.t. shifts and reallocations of individual Dirac masses, and iii) show that these linear stability conditions implies local non-linear stability. Finally, we show that for regular repulsive interaction potential $W_{\varepsilon}$ converging to a singular repulsive interaction potential $W$, the Dirac-type stationary states $\bar{\rho}_{\varepsilon}$ approximate weakly a unique stationary state $\bar{\rho} \in L^{\infty}$. We illustrate our results with numerical examples.
\end{abstract}

\section{INTRODUCTION}

In this paper, we study the following non-local interaction equation:

$$
\partial_{t} \rho=\nabla_{x} \cdot\left(\rho \nabla_{x}[W * \rho+V]\right),
$$

where $\rho(t, x)$ denotes a density of particles/individuals at position $x \in \mathbb{R}^{d}$ and time $t \geq 0$ subject to an even interaction potential $W(x)=W(-x)$ and an external potential $V(x)$.

Non-local equations like (1) have recently obtained focused attention as macroscopic- or continuum models for microscopic- or particle-discrete processes. Examples for such models are various: Simplified inelastic models for granular media are described by convex attractive potentials [BCP, Tos, CMV], collective behaviour of individuals, such as swarming or chemotaxis gives rise to a variety of continuum models [MEK, TB, TBL, MCO, CH, BCM00, BCM07, BDP, CR, Pat, BDP]. In [KPSV, PSV], (1) is used to model the network of F-actin filaments in the cellular cytoskeleton. Finally, related models can be found in opinion dynamics [BKR] or Lennard-Jones type potentials used in crystallisation [The].

A main feature of models like eq. (1) lies in the concentration of solutions towards measures for aggregative interaction potential. For regular $C^{2}$-potentials

2000 Mathematics Subject Classification. 35B35, 35B20, 35Q80 .

Key words and phrases. non-local interaction equation, double-well potential, stability analysis, numerical simulation. 
as in [CMV, KPSV, PSV, CT] aggregation towards measures occurs as time goes to infinity. More singular attractive interaction potentials like $W(x) \sim|x|^{1+\alpha}$ with $0 \leq \alpha<1$, will lead to concentration of solutions to Dirac measures within finite time [BL, BCL, Lau, BB] or even collapse to a unique Dirac mass within finite time [CDFLS].

Adding a diffusion term to eq. (1) can prevent blow-up of solutions and may produce smoothed blow-up profiles for attractive interaction potentials (see e.g. [BDiF, CMV]). However, the famous Patlak-Keller-Segel model of chemotaxis shows that strongly singular attractive interactions, such as $W(x)=-\frac{1}{2 \pi} \log |x|$ in dimension 2, are able to counterbalance diffusion. More precisely for the 2D Patlak-Keller-Segel model, there exists a threshold of critical initial mass, which decides between global solutions and blow-up within finite time (see e.g. $[\mathrm{BDP}, \mathrm{BCC}]$ and the references therein and $[\mathrm{BL}]$ for chemotaxis without diffusion and various type of attractive singularities).

In this article we shall focus the one-dimensional case

$$
\partial_{t} \rho=\partial_{x}\left(\rho \partial_{x}(W * \rho)+V\right), \quad x \in \mathbb{R} .
$$

Note that $(2)$ conserves the total mass $\int_{\mathbb{R}} d \rho(x)=1$, which w.l.o.g. shall be normalised to one. Thus, the solution $\rho(t, x)$ can be interpreted as a probability density and a change of variables introducing the pseudo-inverse of the distribution function $\int_{-\infty}^{x} d \rho$, i.e.

$$
u(z)=\inf \left\{x \in \mathbb{R}: \int_{(-\infty, x]} d \rho(x)>z\right\} \quad z \in[0,1]
$$

transforms eq. (2) for non-negative measure solutions $\rho(t, x)$ into the following integral equation for non-decreasing functions $u(t, z)$ (see [LT, CT, BDiF])

$$
\partial_{t} u(t, z)=\int_{0}^{1} W^{\prime}(u(\xi)-u(z)) d \xi-V^{\prime}(u(z)), \quad \forall z \in[0,1] .
$$

Indeed, eq. (3) has, for instance, the advantage that atomic parts of measure solutions $\rho(t, x)$ are transformed into constant parts of the pseudo-inverse $u(t, z)$. In order to verify eq. (3), note the useful change of variable $\int_{\mathbb{R}} g(x) d \rho(x)=$ $\int_{0}^{1} g(u(\xi)) d \xi$, which holds for any $g \in L^{1}(\operatorname{supp}(\rho))$.

Moreover, in the absence of a confining potential $V=0$, the centre of mass $\int_{\mathbb{R}} x d \rho(t, x)$, or equivalently, the mass $\int_{0}^{1} u(t, z) d z$ is preserved by (2) or eq. (3), respectively :

$$
V=0 \quad \Rightarrow \quad \frac{d}{d t} \int_{\mathbb{R}} x d \rho(t, x)=0, \quad \frac{d}{d t} \int_{0}^{1} u(t, z) d z=0,
$$

which is a direct consequence of $W^{\prime}$ being anti-symmetric.

The main objective of this article is the stability of stationary states of (2). Previous stability results showed for convex attractive interaction potentials that solutions converge to a single Dirac mass as stationary states (see e.g. [CMV, $\mathrm{BDiF}, \mathrm{BCP}, \mathrm{CDFLS}])$. 
However, stationary states consisting of several Dirac masses can be found, for instance, for locally repulsive double-well potential (see [KPSV, PSV] for a model of the two alignment directions of actin-filaments in the cellular cytoskeleton).

As an example, consider a smooth double-well interaction potential $W$ with a local maximum at $x=0$ and a local minimum at $x=x_{1}>0$ in the absence of an external potential, i.e. $V=0$. Thus by (4), the (centre of) mass $\int_{0}^{1} u(z) d z=0$ is conserved in time.

First, we observe that the constant solution $\bar{u}=0$ is obviously (due to $W^{\prime}(0)=$ 0 ) a stationary state of (3) and corresponds to a single Dirac mass $\rho(x)=\delta_{0}(x)$ at $x=0$. Moreover, $\bar{u}=0$ is linear unstable w.r.t. mass-preserving perturbations $u=\bar{u}+v(z)$ with $\int_{0}^{1} v(z) d z=0$ :

$$
\partial_{t} v(t, z)=\int_{0}^{1} W^{\prime \prime}(0)(v(\xi)-v(z)) d \xi=-W^{\prime \prime}(0) v(z),
$$

and $-W^{\prime \prime}(0)>0$ as the double-well is locally repulsive.

In fact, the aggregative/confining effect of the double-well potential over large distances is first seen on the following family of steady states with two Dirac masses $\bar{\rho}(x)$ or monotone increasing two-valued step-functions $\bar{u}(z)$ :

$$
\begin{aligned}
& \bar{u}\left(z, z_{0}\right)=\left\{\begin{array}{rl}
-\left(1-z_{0}\right) x_{1} & z<z_{0}, \\
z_{0} x_{1} & z>z_{0},
\end{array}\right. \\
& \bar{\rho}\left(x, z_{0}\right)=z_{0} \delta_{-\left(1-z_{0}\right) x_{1}}+\left(1-z_{0}\right) \delta_{z_{0} x_{1}} .
\end{aligned}
$$

where the parameter $z_{0} \in(0,1)$ denotes the jump-point and the parameter of mass distribution, respectively. Note that the two Dirac masses of (5) are set apart by the same distance $x_{1}$ as the two extremal points of $W$.

Linear stability analysis will show in section 3 that if the repulsive concavity is dominated by the aggregating convexity (i.e. $-W^{\prime \prime}(0)<W^{\prime \prime}\left(x_{1}\right)$ ), the two-Dirac stationary state (5) are linearly stable on an open interval of the parameter of mass-distribution $z_{0}$ :

$$
-W^{\prime \prime}(0)<W^{\prime \prime}\left(x_{1}\right) \quad \Rightarrow \quad \text { linear stability of }(5) \quad \forall z_{0} \in\left(1-z_{0}^{*}, z_{0}^{*}\right),
$$

where $z_{0}^{*}:=\frac{W^{\prime \prime}\left(x_{1}\right)}{W^{\prime \prime}\left(x_{1}\right)+W^{\prime \prime}(0)}>\frac{1}{2}$. This means that linear stability holds if and only if the mass distribution is not too asymmetric.

For the opposite case with dominating repulsion $-W^{\prime \prime}(0)>W^{\prime \prime}\left(x_{1}\right)$, the question of stable stationary states arises and we conjecture at this point the existence of stable states with more than two Dirac masses.

It is indeed not very surprising that local repulsion has a distributive effect on the stationary states. As example, consider the extreme limit $W(x) \rightarrow \delta_{0}(x)$, in which the non-local term in eq. (2) converges formally towards a quadratic diffusion term, which is expected to render stationary states continuous. 
While we will not consider diffusion terms in this paper, we shall nevertheless point out the diffusion-like effects of local singular repulsive interaction potentials. Examples for such singular repulsive potentials appear, for instance, in swarming models, like the attractive-repulsive Morse potential $W(x)=-C_{a} e^{-|x| / l_{a}}+$ $C_{r} e^{-|x| / l_{r}}$. For repulsive potentials with modulus like singularity at $x=0$, solutions subject to bounded initial data are no longer expected to convergence to measures, but to remain bounded (see [Rao2]).

Throughout this article we shall suppose the following assumptions on the symmetric interaction potential $W$ and on the confining potential $V$ :

\section{Regularity and symmetry assumption}

$$
W(x)=W(-x) \in C^{2, \alpha}(\mathbb{R}), \quad V(x) \in C^{2, \alpha}(\mathbb{R}),
$$

for a Hölder exponent $0<\alpha \leq 1$.

The existence theory of (2) constructs probability measures as solutions via limits of the Jordan-Kinderlehrer-Otto scheme after interpreting (2) as a gradient flow on Wasserstein spaces. In $[\mathrm{BDiF}]$, this was done essentially under the assumption (6). In [CDFLS], the existence theory was generalised to any dimension and singular attractive interaction potentials $W$, which are (amongst other assumptions) $\lambda$-convex (i.e. $W-\frac{\lambda}{2} x^{2}$ is convex for a $\lambda<0$ ).

In this article, we shall only consider compactly supported solutions $\rho(t, x)$. In section 2 , we will recall uniform-in-time propagation of compact support of solutions of (2) under one of the following sufficient conditions:

\section{Initial support and confinement assumptions}

$$
\begin{aligned}
& \rho_{i n}(x) \in M^{1}(\mathbb{R}), \quad \operatorname{supp}\left(\rho_{i n}\right) \subset[-C, C] \quad \text { for a } C<\infty, \\
& V \neq 0: \quad \exists C_{1}>C:\left\|W^{\prime}\right\|_{L^{\infty}\left(\left[-2 C_{1}, 2 C_{1}\right]\right)}<\min \left\{V^{\prime}\left(C_{1}\right),-V^{\prime}\left(-C_{1}\right)\right\},
\end{aligned}
$$

or

$$
V=0: \quad \exists C_{1}, C_{2}>C: W^{\prime}(-x) \leq-C_{2} x, W^{\prime}(x) \geq C_{2} x, \quad \forall x \geq C_{1},
$$

Note that uniform compact support follows under condition $(7 \mathrm{~b})$ since the external potential $V$ confines the interaction potential $W$, while the condition (7c) ensures a "self-confining" interaction potential $W$ in the absence of an external potential $V=0$.

In section 2, we shall also recall from [Rao2] that stationary states of (2) for analytic potentials $V$ and $W$ satisfying one of the conditions (7) are always a finite sum of Dirac masses. Moreover, for less regular interaction potentials $W \in C^{2}$ it was shown in [Rao2] that stationary states $\bar{\rho}$ whose support contains an accumulation point can not have a spectral gap in $L^{1}$ for the linearised equation. Hence, asymptotically stable stationary states of (2) under the assumptions (6) and (7) are necessarily finite sums of Dirac masses. 
The main findings of this paper are organised in the following way:

Proposition 2.4 in subsection 2.1 provides a criterion for a given sum of Dirac masses to be a stationary state of (2) and (3), respectively.

In section 3 , we investigate the stability of a stationary state consisting of a finite sum of Dirac masses. First, we present the above mentioned linear stability analysis of the two Dirac stationary state (5), which serves to identify two eigenspaces representing shifts and reallocations of individual Diracs to decide about linear stability. Corresponding to these eigenspaces, Proposition 3.1 states two necessary conditions for linear stability and Theorem 3.1 proves for regular interaction potentials that these linear stability conditions imply indeed the local non-linear stability of the stationary states under small Wasserstein $W_{\infty^{-}}$ perturbations (i.e. $W_{\infty}(\rho, \bar{\rho})=\|u-\bar{u}\|_{\infty}$ is small enough). We shall also show related numerical simulations.

In section 4 , we show that regular repulsive-aggregative interaction potentials may have stable stationary states consisting of arbitrarily many Dirac masses, which converges weakly towards a continuous stationary state if the repulsive part becomes singular repulsive.

More precisely, we consider smoothed, locally repulsive interaction potentials $W_{\varepsilon}(x)=x^{2}-|x|_{\varepsilon}$ approximating the singular, locally repulsive potential $W(x)=$ $x^{2}-|x|$ as $\varepsilon \rightarrow 0$ and calculate explicitely how a corresponding family of nonunique stable stationary states consisting of an increasing sum of Dirac masses converge weakly towards the unique bounded stationary state of the limiting potential $W$. In Proposition 4.1 we prove this weak limit rigorously for a strictly convex potential $V$ and $W_{\varepsilon}(x)=-|x|_{\varepsilon} \rightarrow W(x)=-|x|$ as $\varepsilon \rightarrow 0$.

Moreover, we illustrate this limit with numerical simulations.

\section{Preliminaries AND Stationary state CONDition}

Throughout this paper, we shall only consider solutions $\rho(t, x)$ with uniformin-time compact support, and thus compactly supported stationary states. The following Proposition states that the confinement conditions (7) on the external potential $V$ and the interaction potential $W$ ensure such solutions.

Proposition 2.1 (Existence and uniform compact support, [BDiF, Rao2]). Let $V$ and $W$ satisfy (6). Then, there exists a unique compactly supported solution $\rho(t, x) \in \operatorname{Lip}_{\text {loc }}\left([0, \infty), \mathcal{P}_{\infty}(\mathbb{R})\right)$ of $(2)$ subject to compactly supported initial data $\rho_{\text {in }} \in M^{1}(\mathbb{R})$ (see $\left.[\mathrm{BDiF}]\right)$.

Let moreover $V$ and $W$ satisfy (7). Then, there exists a constant $C$ such that for all times $t \geq 0$ :

$$
\operatorname{supp}(\rho(t, \cdot)) \subset[-C, C], \quad t \geq 0 .
$$

The following proposition identifies the stationary states of (2) for analytical potentials $V, W$ as finite sums of Dirac masses. 
Proposition 2.2 (Analytic potentials have sums of Dirac masses as stationary state, [Rao2]). Assume that $W$ and $V$ are analytical and satisfy (7). Then, every steady state solution of (2) with bounded support is a finite sum of Dirac masses: $\bar{\rho}=\sum_{i=1}^{n} \rho_{i} \delta_{u_{i}}$ with $\rho_{i}>0$ and $\sum_{i=1}^{n} \rho_{i}=1$.

Proof of Proposition 2.2. For the sake of the reader, we recall the proof of [Rao2]. For a stationary solution $\bar{\rho}$ of (2), or equally for a steady state $\bar{u}$ of (3), we have for $z \in[0,1]$,

$$
0=\int_{0}^{1} W^{\prime}(\bar{u}(\xi)-\bar{u}(z)) d \xi-V^{\prime}(u(z))=-\left(W^{\prime} * \bar{\rho}+V^{\prime}\right)(\bar{u}(z))
$$

and the analytic function $W^{\prime} * \bar{\rho}(x)+V^{\prime}(x)=0$ equals zero for any $x=\bar{u}(z) \in$ $\operatorname{supp}(\bar{\rho})$ on the support of $\bar{\rho}$. Thus, if $\operatorname{supp}(\bar{\rho})$ has an accumulation point, then $W^{\prime} * \bar{\rho}(x)+V^{\prime}(x) \equiv 0$ is constant zero. This is in contradiction to the compact support conditions $(7 \mathrm{~b})$ and $(7 \mathrm{c})$. We conclude that the $\operatorname{support} \operatorname{supp}(\bar{\rho})$ is therefore a finite set of points.

Note that the interaction potential $W(x):=\operatorname{dist}(x,[-1,1])$, where dist denotes a $C^{\infty}$-version of the distance towards the interval $[-1,1]$ admits the $L^{1}(\mathbb{R})$ steady state $\bar{\rho}=\mathbb{1}_{\left[-\frac{1}{2}, \frac{1}{2}\right]}$ and thus shows that such a strong result can no longer be expected for non-analytic potentials.

Nevertheless, the following proposition states that steady states which are strictly linearly stable are necessarily sums of Dirac masses:

Proposition 2.3 (Sums of Dirac masses are the only asymptotically stable stationary states for regular potentials, [Rao2]). Let $V, W$ satisfy (6). Let $\bar{\rho}$ be a steady state of (2), and $\bar{u}$ being its pseudo-inverse. If $\bar{\rho}$ is such that $\operatorname{supp}(\bar{\rho})$ has an accumulation point, then the pseudo-inverse equation (3) linearised around $\bar{u}$ in $L^{1}$ has no spectral gap.

2.1. Sums of Dirac masses as stationary states. In this subsection, Proposition 2.4 provides a criterion for finite sum of $n \in \mathbb{N}$ Dirac masses with normalised mass to be a stationary state $\bar{\rho}(x)$ of $(2)$ :

$$
\bar{\rho}(x)=\sum_{i=1}^{n} \rho_{i} \delta_{u_{i}}(x), \quad \sum_{i=1}^{n} \rho_{i}=1, \rho_{i}>0,
$$

which corresponds to increasing stepfunction $\bar{u}(z)$ as stationary states of (3)

$$
\bar{u}(z)=\sum_{i=1}^{n} u_{i} \mathbb{1}_{I_{i}}, \quad I_{i}=\left[\sum_{j<i} \rho_{j}, \sum_{j \leq i} \rho_{j}\right), \quad\left|I_{i}\right|=\rho_{i} .
$$

Proposition 2.4 (Stationary condition for sums of Dirac masses). Assume $V$, $W \in C^{1}(\mathbb{R})$. For a given integer $n \in \mathbb{N}$, a non-negative measure $\bar{\rho}(x)$ as in $(8)$ or an increasing stepfunction $\bar{u}(z)$ as in (9) constitutes a compactly supported 
stationary state of eq. (2) or eq. (3), respectively, if and only if the following condition holds

$$
\sum_{j=1}^{n} W^{\prime}\left(u_{j}-u_{i}\right) \rho_{j}=V^{\prime}\left(u_{i}\right), \quad i=1, \ldots, n .
$$

Proof of Proposition 2.4. A stepfunction $\bar{u}=\sum_{i=1}^{n} u_{i} \mathbb{1}_{I_{i}}$ with $\left|I_{i}\right|=\rho_{i}$ is a steady-state of (3) iff for all $z \in I_{i}, i=1, \ldots, n$ :

$$
0=\int_{0}^{1} W^{\prime}\left(\bar{u}(\xi)-u_{i}\right) d \xi-V^{\prime}\left(u_{i}\right)=\sum_{j=1}^{n} \rho_{j} W^{\prime}\left(u_{j}-u_{i}\right)-V^{\prime}\left(u_{i}\right), \quad z \in I_{i},
$$

which shows (10).

Example 2.1 (Examples of stationary states). If $V=0$, the condition (10) in the case $n=3$ of three Dirac masses constitutes the vector product

$$
\left(\begin{array}{c}
-W^{\prime}\left(u_{3}-u_{2}\right) \\
W^{\prime}\left(u_{3}-u_{1}\right) \\
-W^{\prime}\left(u_{2}-u_{1}\right)
\end{array}\right) \times\left(\begin{array}{c}
\rho_{1} \\
\rho_{2} \\
\rho_{3}
\end{array}\right)=0
$$

which is satisfied by a positive, normalised vector of masses

$$
\left(\rho_{1}, \rho_{2}, \rho_{3}\right)=\frac{\left(-W^{\prime}\left(u_{3}-u_{2}\right), W^{\prime}\left(u_{3}-u_{1}\right),-W^{\prime}\left(u_{2}-u_{1}\right)\right)}{-W^{\prime}\left(u_{3}-u_{2}\right)+W^{\prime}\left(u_{3}-u_{1}\right)-W^{\prime}\left(u_{2}-u_{1}\right)}
$$

if and only if $\operatorname{sign}\left(-W^{\prime}\left(u_{3}-u_{2}\right)\right)=\operatorname{sign}\left(-W^{\prime}\left(u_{2}-u_{1}\right)\right)=\operatorname{sign}\left(W^{\prime}\left(u_{3}-u_{1}\right)\right)$.

For a double-well potential $W(x)$ as mentioned in the introduction (see also (12)) below), this is equivalent to choosing three values $u_{i}$ such that $0<u_{2}-$ $u_{1}, u_{3}-u_{2}<x_{1}$ and $u_{3}-u_{1}>x_{1}$. Thus, there exists a family of stationary states spanned by two of the three values $u_{i}$ as parameters (with the third value determined by $\sum_{i=1}^{3} \rho_{i} u_{i}=0$ due to (4) as $\left.V=0\right)$. In contrast to the two Dirac stationary states $(5)$ (for which the parameter $z_{0} \in(0,1)$ determines equally the $u_{i}$ values and the masses $\rho_{i}$ ) depend solutions of (11) on the monotonicity of $W^{\prime}$ in order to find either zero, a unique, several, or even infinitely many values $u_{i}$ for given three masses $\rho_{i}$. However, for generic $W^{\prime}$ we can expect a unique vector of values $u_{i}$.

For odd numbers of Dirac masses, the skew-symmetric matrix $W^{\prime}\left(u_{i}-u_{j}\right)$ has (at least) one zero-eigenvalue as the case $n=3$. Thus, similar families of stationary states will exist for non-trivial matrices $W^{\prime}\left(u_{i}-u_{j}\right)$. Hence, without external potential $V=0$, we have to expect stationary states, which consists out of arbitrarily many Dirac masses. In section 4 we shall see that these stationary states can be stable.

On the other hand, for even numbers of Dirac masses, the skew-symmetric matrices $W^{\prime}\left(u_{i}-u_{j}\right)$ are generically invertible for potential with proper monotonicity $W^{\prime}$. Thus, for given external potential $V(x)$, we expect steady states 
satisfying:

$$
\rho_{j}=\sum_{i=1}^{n}\left(W^{\prime}\left(u_{j}-u_{i}\right)\right)^{-1} V^{\prime}\left(u_{i}\right), \quad j=1 \ldots n,
$$

provided the $\rho_{j}$ are positive.

\section{LOCAL STABILITY OF DisCRETE STATIONARY STATES}

In this section, we will study the stability of stationary states consiting of a finite sum of Dirac masses.

We recall the example mentioned in the introduction of a smooth doublewell interaction potential $W$ with a single local maximum at $x=0$ and a local minimum at $x= \pm x_{1}$ for a $x_{1}>0$ :

$$
\beta:=-W^{\prime \prime}(0)>0, \quad W^{\prime}(0)=0, \quad \alpha:=W^{\prime \prime}\left( \pm x_{1}\right)>0, \quad W^{\prime}\left( \pm x_{1}\right)=0 .
$$

Due to the absence of an external potential $V=0$ the conservation law (4) holds and the (centre of) mass $\int_{0}^{1} u(z) d z=0$ is conserved in time.

In order check for stability of the two Dirac steady states (5), i.e.

$$
\bar{u}\left(z, z_{0}\right)=\left\{\begin{array}{rl}
-\left(1-z_{0}\right) x_{1} & z<z_{0} \\
z_{0} x_{1} & z>z_{0}
\end{array}\right.
$$

we linearise around $\bar{u}$ and apply the exponential ansatz $v(z)=e^{\lambda t} \phi(z)$ for mass preserving perturbations $\int_{0}^{1} v(z) d z=0=\int_{0}^{1} \phi(z) d z=0$, which yields the following mixed local/non-local eigenproblem

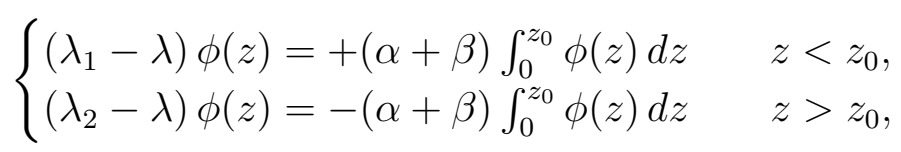

where $\lambda_{1}$ and $\lambda_{2}$ are the following convex combinations of $\alpha$ and $\beta$

$$
\lambda_{1}:=z_{0} \beta-\left(1-z_{0}\right) \alpha, \quad \lambda_{2}:=\left(1-z_{0}\right) \beta-z_{0} \alpha .
$$

We identify two eigenspaces depending on the left hand side of (13) :

Shifts: In case that $\lambda_{1} \neq \lambda \neq \lambda_{2}$ the eqs. (13) show $\phi$ to be piecewise constant and a simple calculation leads to the stable eigenmodes

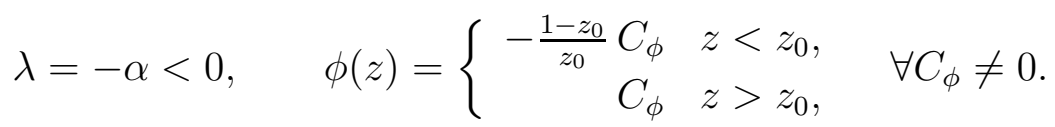

Note that the corresponding eigenspace consists of mass-preserving spatial shifts of the two Dirac masses. Stability w.r.t shifts is then due to the stabilising interaction between the two aggregates as the interaction potential $W$ is aggregative at the distance $x_{1}$ with $\alpha=W^{\prime \prime}\left( \pm x_{1}\right)>0$. 
Reallocations: In case that $\lambda_{1}=\lambda \neq \lambda_{2}$, we have $\int_{0}^{z_{0}} \phi(z) d z=0$ by the first eq. in (13) and $\phi=0$ for $z>z_{0}$ by the second eq. in (13), which yields the eigenmodes

$$
\lambda=z_{0} \beta-\left(1-z_{0}\right) \alpha, \quad \phi(z)=\left\{\begin{array}{rl}
\varphi_{l}(z) & z<z_{0}, \\
0 & z>z_{0},
\end{array} \quad \forall \varphi_{l}(z): \int_{0}^{z_{0}} \varphi_{l} d z=0 .\right.
$$

By the symmetry $z_{0} \leftrightarrow\left(1-z_{0}\right)$ in (13) we find mirrored eigenmodes for $\lambda_{2}=\lambda \neq \lambda_{1}$. If, especially $\lambda_{1}=\lambda=\lambda_{2}$ and, thus, $z_{0}=\frac{1}{2}$, we obtain

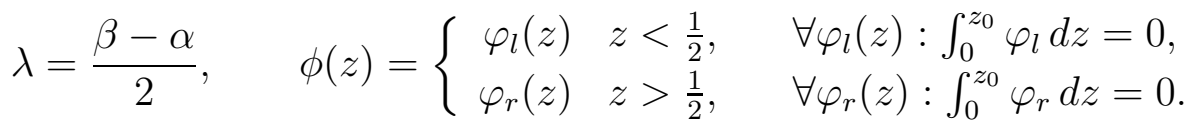

All these eigenspaces represent mass-preserving reallocations of one (or both) Dirac masses. Note that a local reallocation does not need to smooth a Dirac mass. Indeed, a simple reallocation consist of splitting a Dirac into two by two local shifts within the intervals $\left(0, z_{0}\right)$ or $\left(z_{0}, 1\right)$.

Stability w.r.t. reallocations for equal mass distribution $z_{0}=\frac{1}{2}$ is given if and only if the short range repulsion is controlled by the long range aggregation, i.e. $\beta<\alpha$. Moreover, for asymmetric mass distribution $z_{0} \neq \frac{1}{2}$, there exists a threshold of maximal asymmetry, where $\lambda_{1}\left(z_{0}^{*}\right)=$ $z_{0}^{*} \beta-\left(1-z_{0}^{*}\right) \alpha=0$.

Altogether for a locally repulsive double-well potential (12) we have linear stability of the steady states (5) with respect to mass-preserving shifts and reallocations provided that $\beta<\alpha$ on an open interval of parameters $z_{0}$ :

$$
\beta<\alpha \Rightarrow \max \left\{\lambda_{1,2}\left(z_{0}\right)\right\}<0 \quad \forall z_{0} \in\left(1-z_{0}^{*}, z_{0}^{*}\right), \quad z_{0}^{*}:=\frac{\alpha}{\alpha+\beta}>\frac{1}{2} .
$$

This is the results stated in the introduction. Here, we complement it with numerical simulations performed using an explicit Euler scheme for the pseudoinverse equation (3). Note that approximating $u(z)$ on $z \in[0,1]$ by piecewise constant step functions on an equidistant grid with $n+1$ grid points (we have used $n=256$ ) is equivalent to a particle method for equation (2), where a measure $\rho(x)$ is approximated by a sum of $n$ Diracs with mass $\frac{1}{n}$. The numerics implemented in Matlab. In order to depict a measure $\rho(x)$, we represent each Dirac mass by a triangle centred at the position $u_{i}$ with basis-length $1 / 90$ and with area equivalent to the mass of the represented Dirac.

Figure 1 shows how the solution of (2) with the symmetric (in the $\rho$ picture) initial data $u_{i n}(z)=0.05(\sin (6 \pi z)+6 \pi z)-C$ with $C$ such that $\int_{0}^{1} u_{i n}(z) d z=0$ (bold line) converges to the stable symmetric two Dirac stationary state $\bar{\rho}=$ $\frac{1}{2} \delta_{-2^{3 / 2}}+\frac{1}{2} \delta_{2^{3 / 2}}$, for the double-well potential $W(x)=x^{4}-x^{2}$. Note that the initial data constitute three smoothed Dirac masses, where the middle one is placed on a unstable stationary position, i.e. at a local maximum of the function $(W * \rho)(x)$ (recall that $\partial_{t} u(t, z)=\left(W^{\prime} * \rho\right)(u(t, z))$. As a consequence, the middle 
smoothed Dirac persists for a rather long time while the attraction exerted from the two outer smoothed Diracs remains symmetrically balanced.

On the other hand, Figure 2 shows (again for $W(x)=x^{4}-x^{2}$ ) how a small Dirac perturbation of an instable two Dirac stationary state (5) is first slowly, later quickly attracted by the opposite Dirac. Note that the amount of the mass which is exchanged in order to reach a stable stationary state will in general depend on the initial perturbation.

3.1. Linear stability conditions for discrete stationary states. We want to study the stability of finite sum of Dirac masses steady states $\bar{\rho}$ as in (8) by showing stability of the pseudo-inverse $\bar{u}$ as in (9).

We will first introduce two conditions of linear stability, which generalises stability w.r.t. shifts and reallocations as in the example above. For $W$ satisfying (6), the linearised eq. (3) for $u=\bar{u}+v$ reads as:

$$
\begin{aligned}
\partial_{t} v(z)= & \int_{0}^{1} W^{\prime \prime}(\bar{u}(\xi)-\bar{u}(z))(v(\xi)-v(z)) d \xi-V^{\prime \prime}(\bar{u}(z)) v(z) \\
= & -\left(\sum_{j=1}^{n} W^{\prime \prime}\left(u_{j}-\bar{u}(z)\right) \rho_{j}+V^{\prime \prime}(\bar{u}(z))\right) v(z) \\
& +\sum_{j=1}^{n} W^{\prime \prime}\left(u_{j}-\bar{u}(z)\right) \int_{I_{j}} v(\xi) d \xi
\end{aligned}
$$

We recall that in the absence of an external potential $V=0$ the conservation law (4) will permit stability only w.r.t. perturbations $v(z)$, which leave the (centre of) mass unchanged $\int_{0}^{1} v(z) d z=0$.

Recalling the above example suggests two eigenspaces to be checked for linear stability: First, all perturbations leading to

$$
\text { Reallocations, i.e. }\left\{v(z): \begin{array}{c}
\int_{I_{i}} v(\xi) d \xi=0, i=1, \ldots, n \\
\text { if } V=0 \text { then } \int_{0}^{1} v(z) d z=0
\end{array}\right\},
$$

and secondly, perturbations yielding to

$$
\text { Shifts, i.e. }\left\{v(z): \begin{array}{c}
v=\sum_{i=1}^{n} v_{i} \mathbb{1}_{I_{i}} \\
\text { if } V=0 \text { then } \sum_{i=1}^{n} v_{i} \rho_{i}=0
\end{array}\right\} .
$$

The following statements of linear stability hold:

Proposition 3.1. Let $V$ and $W$ satisfy (6). Then, for stationary states $\bar{\rho}=$ $\sum_{i=1}^{n} \rho_{i} \delta_{u_{i}}$ as given in (8), or $\bar{u}=\sum_{i=1}^{n} u_{i} \mathbb{1}_{I_{i}}$ as given in (9), we have:

Linear stability with respect to reallocations: if and only if

$$
0<m_{i}:=\sum_{j=1}^{n} W^{\prime \prime}\left(u_{j}-u_{i}\right) \rho_{j}+V^{\prime \prime}\left(u_{i}\right) \quad \forall i=1, \ldots, n .
$$



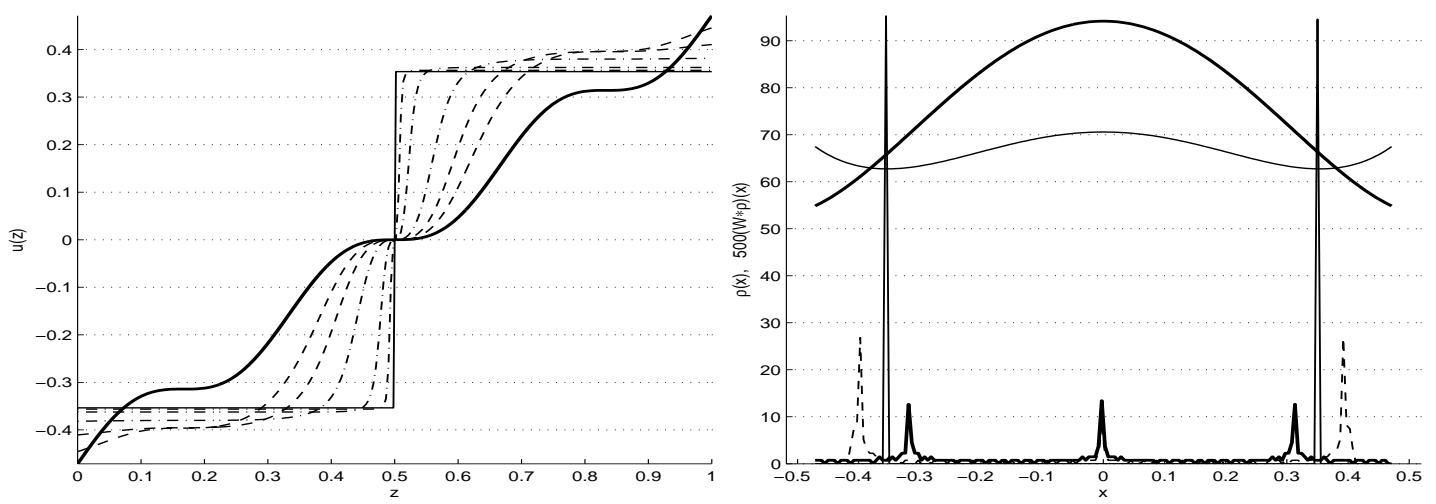

Figure 1. Convergence towards a stable, symmetric two Dirac stationary state (5) for the doublewell potential $W(x)=x^{4}-x^{2}$. The left image plots $u(t, z)$ at time $t=0$ (initial data, bold line), $t=1,2$ (dashed lines), $t=4,6,9,15$ (dash-dotted lines), and $t=$ 25 (stable stationary state, solid line). The right image plots the measures $\rho(t, x)$ and the amplified potential $500(W * \rho)(x)$ at the times $t=0$ (bold lines) and $t=25$ (solid line) as well as $\rho(t, x)$ at time $t=2$ (dashed line).
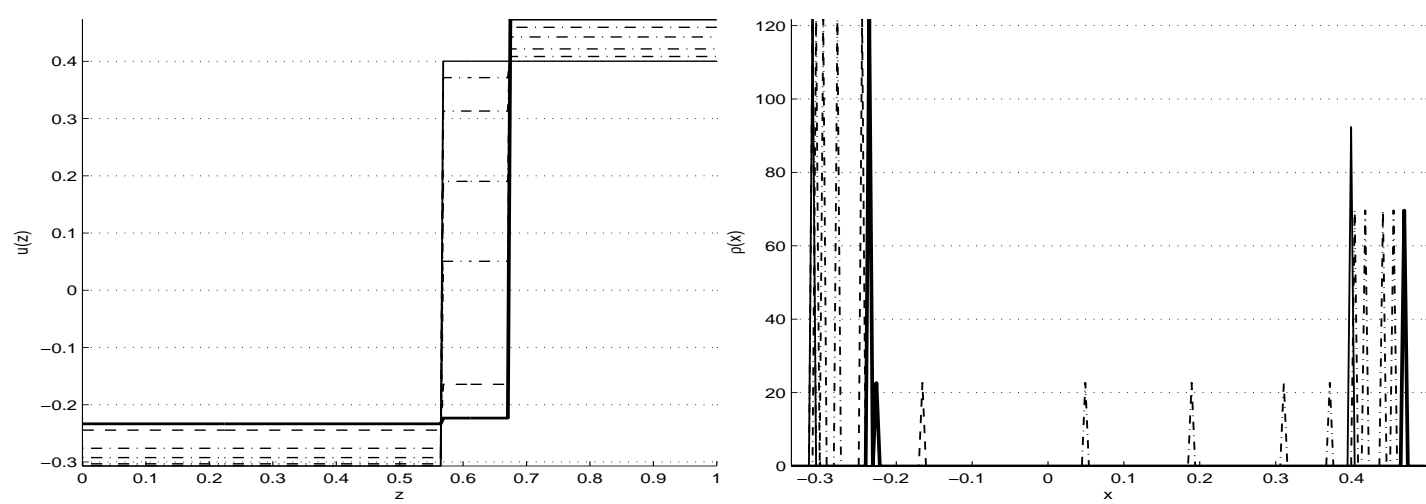

Figure 2. Perturbation of a two Dirac stationary state (5) with slightly unstable mass distribution for the doublewell potential $W(x)=x^{4}-x^{2}$ (with slightly meaning $z_{0}=0.67$ compared to the critical value $z_{0}^{*}=\frac{2}{3}$ ) leads to exchange of mass and convergence towards a stationary state with stable mass distribution. The left image plots $u(t, z)$ at time $t=0$ (initial data, bold line), $t=25$ (dashed line) $t=30,31,32,33$ (fast exchange of mass, dash-dotted lines), and $t=40$ (stable stationary state, solid line). The right image plots the measures $\rho(t, x)$ at times $t=0$ (two Dirac masses plus Dirac perturbation close to the left Dirac, bold line), $t=25$ (dashed line), $t=30,31,32,33$ (fast exchange of mass, dash-dotted lines) and $t=40$ (stable stationary state, solid line). 
Linear stability with respect to shifts: if and only if the matrix $M$

$$
M:=\operatorname{diag}\left(m_{i}\right)-\left(\rho_{i} W^{\prime \prime}\left(u_{j}-u_{i}\right)\right)
$$

has a strictly positive spectrum $\sigma(M)$ in the sense that for some $\nu>0$ either

(SS1) $\quad V \neq 0: \quad \sigma(M) \subset\{z \in \mathbb{C}: \mathcal{R} e(z)>\nu\}$.

or, in the case $V=0$, the spectrum $\sigma\left(\left.M\right|_{H}\right)$ of $M$ restricted onto the hyperspace $H=\left\{\left(w_{i}\right)_{i=1, \ldots, n}: \sum_{i=1}^{n} w_{i}=0\right\}$ is strictly positive

(SS2) $\quad V=0: \quad \sigma\left(\left.M\right|_{H}\right) \subset\{z \in \mathbb{C}: \mathcal{R} e(z)>\nu\}$.

Remark 3.1. Note that in the following subsection we will show that the conditions (SR) and (SS1) or (SS2) imply also local non-linear stability in a sense as defined below.

Proof of Proposition 3.1. When restricted to reallocating perturbations (15) the second term of the linearised equation (14) vanishes and stability is obviously equivalent to $m_{i}>0$ for all $i=1, \ldots, n$.

To show stability with respect to shifts, we have to control the second term of the linearised equation (14) proportional to the vector $\int_{I_{i}} v(z) d z$. Therefore, we integrate over $I_{i}$

$$
\frac{d}{d t}\left(\int_{I_{i}} v(z) d z\right)=-M\left(\int_{I_{i}} v(\xi) d \xi\right), \quad i=1, \ldots, n,
$$

which yields directly the stability condition $(\mathbf{S S 1})$. In the case $V=0$, the conservation law $\int_{0}^{1} v(\xi) d \xi=0$ allows to eliminate one component $\int_{I_{i}} v(z) d z$, which leads to the stability condition (SS2).

Remark 3.2. Notice that if (SS2) is satisfied, $H$ is the eigenspace of $M$ associated to the eigenvalue 0 .

Example 3.1. Following up the above example of two Dirac masses stationary states $\bar{\rho}=z_{0} \delta_{-\left(1-z_{0}\right) x_{1}}+\left(1-z_{0}\right) \delta_{z_{0} x_{1}}$, the stationary condition (10)

$$
\left(\begin{array}{cc}
0 & -W^{\prime}\left(x_{1}\right) \\
W^{\prime}\left(x_{1}\right) & 0
\end{array}\right) \cdot\left(\begin{array}{c}
z_{0} \\
1-z_{0}
\end{array}\right)=0 .
$$

holds iff $W^{\prime}\left(x_{1}\right)=0$. Linear stability w.r.t. reallocations $(\mathbf{S R})$ requires

$$
0<m_{i}=\sum_{j=1}^{n} W^{\prime \prime}\left(u_{j}-u_{i}\right) \rho_{j}= \begin{cases}-\beta z_{0}+\alpha\left(1-z_{0}\right)>0 & i=1, \\ \alpha z_{0}-\beta\left(1-z_{0}\right)>0 & i=2,\end{cases}
$$

which is satisfied for $\frac{\beta}{\alpha+\beta}<z_{0}<\frac{\alpha}{\alpha+\beta}$ and recovers the linear stability result at the beginning of this section. Moreover, linear stability w.r.t. local shifts, i.e. (SS2) 
holds always true as:

$$
M=\left(\begin{array}{cc}
\alpha\left(1-z_{0}\right) & -\alpha z_{0} \\
-\alpha\left(1-z_{0}\right) & +\alpha z_{0}
\end{array}\right) \Rightarrow M \cdot\left(\begin{array}{c}
v \\
-v
\end{array}\right)=\alpha\left(\begin{array}{c}
v \\
-v
\end{array}\right)
$$

on the hyperspace $\left\{\left(v_{1}, v_{2}\right): v_{1}+v_{2}=0\right\}$,

Example 3.2 (Stability of three Dirac masses). There is no simple statement on the stability of stationary states consisting of the three Dirac masses (11) possible. Indeed, asking for stability w.r.t. reallocations, it seems that the sign of the $m_{i}$

$$
m_{i}=\frac{\left.-W^{\prime \prime}\left(u_{1}-u_{i}\right) W^{\prime}\left(u_{3}-u_{2}\right)+W^{\prime \prime}\left(u_{2}-u_{i}\right) W^{\prime}\left(u_{3}-u_{1}\right)-W^{\prime \prime}\left(u_{3}-u_{i}\right) W^{\prime}\left(u_{2}-u_{1}\right)\right)}{-W^{\prime}\left(u_{3}-u_{2}\right)+W^{\prime}\left(u_{3}-u_{1}\right)-W^{\prime}\left(u_{2}-u_{1}\right)}
$$

can be tuned by modifying $W^{\prime \prime}\left(u_{j}-u_{i}\right)$ while keeping $W^{\prime}\left(u_{j}-u_{i}\right)$ fixed.

The possible stability of the three Dirac stationary states is important for models using interaction potentials. For instance, one might intuitively expect that stable stationary states of a double-well potential (12) should consist of two Dirac masses. However, the above formula for the $m_{i}$ suggests and section 4 will show that there are stable three (and more) Dirac stationary states, which are even locally non-linear stable in the sense of the following section. This raises the question of the robustness versus the flexibility of stationary states under quasistationary model variations. This might be relevant, for instance, when modelling the dynamics of the cytoskeleton [KPSV, PSV].

3.2. Local non-linear stability without mass exchange. In this section, we prove local non-linear stability of stationary states consisting of finitely many Dirac masses $\bar{\rho}=\sum_{i=1}^{n} \rho_{i} \delta_{u_{i}}$ as given in (8) under small Wasserstein $W_{\infty}$-perturbations of.

More precisely, the proof shows equivalently the local non-linear stability of stationary states $\bar{u}=\sum_{i=1}^{n} u_{i} \mathbb{1}_{I_{i}}$ as in (9) of the pseudo-inverse equation (3) under small $L^{\infty}$-perturbations $u=\bar{u}+v$ of $\bar{u}$ such that

$$
\|v\|_{L^{\infty}}([0,1]) \leq \varepsilon
$$

for a $\varepsilon>0$. Such a perturbation $v(z)$ is equivalent to small Wasserstein $W_{\infty}$-differences $W_{\infty}(\bar{\rho}, \rho) \leq \varepsilon$ for two probability measure $\rho$ and $\bar{\rho}$, that is:

$$
\left\{\begin{array}{l}
\left.(\rho-\bar{\rho})\right|_{\mathbb{R} / \cup_{i=1}^{n}\left[u_{i}-\varepsilon, u_{i}+\varepsilon\right]}=0, \\
\int_{\left[u_{i}-\varepsilon, u_{i}+\varepsilon\right]} d \rho=\int_{\left[u_{i}-\varepsilon, u_{i}+\varepsilon\right]} d \bar{\rho}, \quad \forall i=1, \ldots, n,
\end{array}\right.
$$

Finally, in the case $V=0$, the conservation of (the centre) of mass (4) restricts additionally the admissible perturbations such that

$$
\int_{0}^{1} v(\xi) d \xi=0, \quad \int_{\mathbb{R}} x d \rho(x)=\int_{\mathbb{R}} x d \bar{\rho}(x) .
$$

Theorem 3.1 (Local non-linear stability). Let $V$ and $W$ satisfy (6) and (7). Assume that a steady-state $\bar{u}=\sum_{i=1}^{n} u_{i} \mathbb{1}_{I_{i}}$ of eq. (3) or $\bar{\rho}=\sum_{i=1}^{n} \rho_{i} \delta_{u_{i}}$ of eq. (2), 
is linear stable w.r.t. reallocations, (i.e. (SR) holds) and shifts (i.e. (SS1) holds or (SS2) holds if $V=0)$.

Then, for initial data $u_{\text {in }}$ satisfying $\left\|u_{i n}-\bar{u}\right\|_{\infty}$ small enough (and preserving the (centre of) mass if $V=0$ ), we have

$$
\|u(t)-\bar{u}\|_{\infty}=W_{\infty}(\rho(t), \bar{\rho}) \leq C\left(1+t^{n-1}\right) e^{-\eta t},
$$

for a constant $C$ and with $\eta:=\min \left\{\nu, m_{1}, \ldots, m_{n}\right\}$ with $\nu$ defined in (SS1) or (SS2), and the $m_{i}$ are defined by (SR).

Remark 3.3. For more general perturbations $\rho_{\text {in }}-\bar{\rho} \in M^{1}(\mathbb{R})$, one can only expect orbital stability. This is currently work in progress in [Rao3].

Remark 3.4. The smallness assumption of Theorem 3.1 on $\|u-\tilde{u}\|_{\infty}$ for two probability measures $\bar{\rho}=\sum_{i=1}^{n} \rho_{i} \delta_{u_{i}}$ and $\tilde{\rho}=\sum_{i=1}^{n} \tilde{\rho}_{i} \delta_{\tilde{u}_{i}}$ is satisfied if

$$
\left|u_{i}-\tilde{u}_{i}\right| \leq \varepsilon,\left|\rho_{i}-\tilde{\rho}_{i}\right| \leq \varepsilon, \quad \forall i=1, \ldots, n .
$$

In order to prove Theorem 3.1, we will need the following technical lemma:

Lemma 3.1. If for all $t \geq 0$ the matrix $N(t) \in L^{\infty}\left(M_{n}(\mathbb{C})\right)$ is upper triangular with $\mathcal{R} e\left(n_{i i}(t)\right) \leq-\eta<0$ for all $i=1, \ldots, n$, then there exist a constant $C>0$ such that

holds for all induced matrix norms.

$$
\left\|e^{\int_{0}^{t} N(s) d s}\right\| \leq C\left(1+t^{n-1}\right) e^{-\eta t}
$$

Proof of Lemma 3.1. We define $w(t):=e^{\int_{0}^{t} N(s) d s} X$ for $X \in \mathbb{C}^{n}$ and $w(t)$ is the solution of the differential equation

$$
\frac{d}{d t} w(t)=N(t) w(t)=\operatorname{diag}\left(n_{i i}(t)\right) w(t)+\left[N(t)-\operatorname{diag}\left(n_{i i}(t)\right)\right] w(t)
$$

subject to the initial condition $w(0)=X$. Then, $w_{i}(t)$ is given by

$$
w_{i}(t)=e^{\int_{0}^{t} n_{i i}(s) d s} w_{i}(0)+\sum_{j=i+1}^{n} \int_{0}^{t} e^{\int_{s}^{t} n_{i i}\left(s^{\prime}\right) d s^{\prime}} n_{i j}(s) w_{j}(s) d s,
$$

and a backward recurrence argument shows that

$$
\left|w_{i}(t)\right| \leq C\left(1+t^{n-i}\right)\|X\|_{\infty} e^{-\eta t} \quad \forall i=1, \ldots, n .
$$

Indeed, (18) holds for $i=n$ and assuming (18) for $i \in\{I+1, \ldots, n\}$ yields

$$
\begin{aligned}
\left|w_{I}(t)\right| & \leq\left|w_{i}(0)\right| e^{-\eta t}+\sum_{j=I+1}^{n} \int_{0}^{t} C\left(1+s^{n-j}\right)\|X\|_{\infty} e^{-\eta s} e^{-\eta(t-s)} d s \\
& \leq\left|w_{i}(0)\right| e^{-\eta t}+C\left(1+t^{n-I-1}\right) t\|X\|_{\infty} e^{-\eta t} \\
& \leq C\left(1+t^{n-I}\right)\|X\|_{\infty} e^{-\eta t},
\end{aligned}
$$

which proves the lemma after using the equivalence of matrix norms.

We now prove Theorem 3.1: 
Proof of Theorem 3.1.

Step 1: We first show an estimate on the vector $\int_{I_{i}} v(t, z) d z$.

Given $V, W \in C^{2, \alpha}$, we Taylor expand eq. (3) point wise around $u=\bar{u}+v$ :

$$
\begin{aligned}
\partial_{t} v(z)= & \int_{0}^{1} W^{\prime \prime}(\bar{u}(\xi)-\bar{u}(z))(v(\xi)-v(z)) d \xi-V^{\prime \prime}(\bar{u}(z)) v(z) \\
& +\int_{0}^{1}\left[W^{\prime \prime}\left(\bar{u}(\xi)-\bar{u}(z)+\theta_{1}(\xi, z)\right)-W^{\prime \prime}(\bar{u}(\xi)-\bar{u}(z))\right](v(\xi)-v(z)) d \xi \\
& +\left[-V^{\prime \prime}\left(\bar{u}(z)+\theta_{2}(z)\right)+V^{\prime \prime}(\bar{u}(z))\right] v(z)
\end{aligned}
$$

where $\left|\theta_{1}(\xi, z)\right| \leq|v(\xi)-v(z)|$ and $\left|\theta_{2}(z)\right| \leq|v(z)|$. For a finite sum of Dirac masses $\bar{u}=\sum_{i=1}^{n} u_{i} \mathbb{1}_{I_{i}}$, we denote $v_{i}(z)=v(z)$ on the intervals $I_{i}$. Then, by the Hölder continuity of $V^{\prime \prime}$ and $W^{\prime \prime}$, we have for $z \in I_{i}$

$$
\begin{aligned}
\partial_{t} v_{i}(z)= & -v_{i}(z)\left(\sum_{j=1}^{n} W^{\prime \prime}\left(u_{j}-u_{i}\right) \rho_{j}+V^{\prime \prime}\left(u_{i}\right)\right) \\
& +\sum_{j=1}^{n} W^{\prime \prime}\left(u_{j}-u_{i}\right) \int_{I_{j}} v_{j}(\xi) d \xi+O\left(\|v\|_{\infty}^{1+\alpha}\right), \quad \text { for } z \in I_{i},
\end{aligned}
$$

and integration over the intervals $I_{i}$ for $i=1, \ldots, n$ yields

$$
\frac{d}{d t}\left(\int_{I_{i}} v_{i}(z) d z\right)=-M\left(\int_{I_{j}} v_{j}(\xi) d \xi\right)+O\left(\|v\|_{\infty}^{1+\alpha}\right),
$$

where $M$ is the matrix defined by (17).

Note that in case $V=0$ the conservation law (4), i.e $\int_{0}^{1} v(z) d z=0$ allows to eliminate, for instance, the component $\int_{I_{n}} v_{n}(z) d z$ in (19) and the condition (SS2) implies that the resulting $n$-1-dimensional matrix has its spectrum included in $\mathbb{R}_{+} \times i \mathbb{R}$, which we shall again denoted by $M$ for the sake of simplicity.

Next, there exist a change of basis in $\mathbb{C}^{n}$ or $\mathbb{C}^{n-1}$, respectively, such that the matrix $M$ is transformed into an upper triangular matrix $\tilde{M}$ in a new basis $\left(\tilde{e}_{i}\right)$, and then,

$$
\mathcal{R} e\left(\tilde{m}_{i i}\right)>\nu>0,
$$

either for $i=1, \ldots, n$ in the case $V \neq 0$ due to condition (SS1) or for $i=$ $1, \ldots, n-1$ in the case $V=0$ due to condition (SS2).

Similarly, we denote by $\tilde{\omega}_{i}(t)$ the vector $\int_{I_{i}} v(t, z) d z$ in this new base. In this new base, the system (19) writes:

$$
\frac{d}{d t}\left(\tilde{\omega}_{i}\right)=-\tilde{M}\left(\tilde{\omega}_{i}\right)+O\left(\|v\|_{\infty}^{1+\alpha}\right) .
$$


Step 2: We show estimates on the $\left|v_{i}\right|$ by multiplying (3) with $\operatorname{sign}\left(v_{i}(z)\right)$. Recalling the vector $0<m_{i}, i=1 \ldots n$ defined in (SR), we calculate

$$
\partial_{t}\left|v_{i}(z)\right|=-m_{i}\left|v_{i}\right|(z)+\operatorname{sign}(v(z)) \sum_{j=1}^{n} W^{\prime \prime}\left(u_{j}-u_{i}\right) \int_{I_{j}} v_{j}(\xi) d \xi+O\left(\|v\|_{\infty}^{1+\alpha}\right)
$$

Altogether, we have for $\Omega(t):=\left(\left|v_{1}\right|, \ldots,\left|v_{n}\right|, \tilde{\omega}_{1}, \ldots, \tilde{\omega}_{n}\right)$ (or $\tilde{\omega}_{n-1}$ if $\left.V=0\right)$ :

$$
\frac{d}{d t} \Omega=N \Omega+O\left(\|\Omega\|^{1+\alpha}\right) \quad \text { with } \quad N(t)=\left(\begin{array}{cc}
-\operatorname{diag}\left(m_{i}\right) & O(1)(t) \\
0 & -\tilde{M}
\end{array}\right),
$$

where thank to (SS1) and (20),

$$
\max \left\{\mathcal{R} e\left(n_{i i}\right)\right\}=\max \left\{-m_{i},-\mathcal{R} e\left(\tilde{m}_{i i}\right)\right\} \leq \max \left\{-m_{i},-\nu\right\}<0,
$$

and $\left|n_{i j}(t)\right|$ is uniformly bounded in time. Moreover, $\Omega(t)$ is given by:

$$
\Omega(t)=e^{\int_{0}^{t} N(s) d s} \Omega(0)+\int_{0}^{t} e^{\int_{s}^{t} N\left(s^{\prime}\right) d s^{\prime}} O\left(\|\Omega\|^{1+\alpha}\right)(s) d s
$$

and lemma 3.1 estimates for the upper triangular matrix $N$ with $\eta:=\max \left\{m_{i}, \nu\right\}$

$$
\left\|e^{\int_{0}^{t} N(s) d s}\right\|_{\infty} \leq C\left(1+t^{n-1}\right) e^{-\eta t}
$$

and further

$$
\begin{aligned}
\|\Omega(t)\|_{\infty} \leq & C\left(1+t^{n-1}\right) e^{-\eta t}\|\Omega(0)\|_{\infty} \\
& +C \int_{0}^{t}\|\Omega\|_{\infty}^{1+\alpha}(s)\left(1+(t-s)^{n-1}\right) e^{-\eta(t-s)} d s .
\end{aligned}
$$

Thus, a Gronwall type estimate (see [Bee]) shows

$$
\|\Omega(t)\|_{\infty} \leq C\left(1+t^{n-1}\right) e^{-\eta t},
$$

for $\|\Omega(0)\|_{\infty}$ small enough which finishes the proof.

\section{TOWARDS SINGULAR REPULSIVE POTENTIALS}

In this section, we show explicitly the weak limit of the stationary states of regular interaction potentials satisfying (6) as they approximate the singular repulsive potential $W(x)=x^{2}-|x|$. More precisely, we consider a family of interaction potentials $W_{\varepsilon}(x)=x^{2}-|x|_{\varepsilon}$, with $|x|_{\varepsilon}$ denoting a evenly smoothed version of the modulus on the interval $(-\varepsilon, \varepsilon)$ for $\varepsilon>0$ :

$$
W_{\varepsilon}(x)=x^{2}-|x|_{\varepsilon}, \quad W_{\varepsilon}^{\prime}(x)=2 x-\operatorname{sign}_{\varepsilon}(x), \quad W_{\varepsilon}^{\prime \prime}(x)=2-2 \delta_{\varepsilon}(0),
$$

where we only assume that

$$
\operatorname{sign}_{\varepsilon}(0)=0, \quad \operatorname{sign}_{\varepsilon}( \pm \varepsilon)= \pm 1, \quad \delta_{\varepsilon}(0)=\frac{1}{\varepsilon} .
$$

We then suppose a stationary state $\bar{u}=\sum_{i=1}^{n} u_{i} \mathbb{1}_{I_{i}}$ with $\left|I_{i}\right|=\rho_{i}$ consisting of $n$ Dirac masses for a $n \in \mathbb{N}$. By Prop. 2.4 these stationary states satisfy the 
condition (10). If we assume that all the Dirac masses are separated by a distance $\max _{i=1, . ., n}\left\{u_{i+1}-u_{i}\right\}>\varepsilon$, it follows that

$$
0=\sum_{j=1}^{n} \rho_{j} W_{\varepsilon}^{\prime}\left(u_{j}-u_{i}\right)=-2 u_{i}+\sum_{j<i} \rho_{j}-\sum_{j>i} \rho_{j},
$$

where we have used that $\sum_{j=1}^{n} \rho_{j}=1$ and $\sum_{j=1}^{n} u_{j} \rho_{j}=0$. Hence, subtracting (22) for indices $i+1$ and $i$ leads to

$$
u_{i+1}-u_{i}=\frac{\rho_{i}+\rho_{i+1}}{2} .
$$

Hence, choosing a vector of masses $\rho_{i}$ with $\frac{\rho_{i}+\rho_{i+1}}{2}>\varepsilon$, we obtain $n-1$ unique relative distances $u_{i+1}-u_{i}$ consistent with the above assumption $\max _{i=1, . ., n}\left\{u_{i+1}-\right.$ $\left.u_{i}\right\}>\varepsilon$. Moreover, the constraint $\sum_{j=1}^{n} u_{j} \rho_{j}=0$ yields, for instance, to a unique value $u_{1}$, and, thus, to a unique vector $u_{i}$ and stationary state of that type. The special case $\rho_{i}=\frac{1}{n}$ shows that $\varepsilon$ has to be chosen smaller than $\frac{1}{n}$ in order to have such a stationary state.

In order to have stable stationary states Prop. 3.1 requires for stability with respect to reallocations (15) the conditions

$$
m_{i}=\sum_{j=1}^{n} \rho_{j} W_{\varepsilon}^{\prime \prime}\left(u_{j}-u_{i}\right)=2-\frac{\rho_{i}}{\varepsilon}>0,
$$

which imply the constraint $\varepsilon>\frac{\rho_{i}}{2}$. Secondly, stability with respect to shifts (16) holds always as the matrix

$$
M=\operatorname{diag}\left(m_{i}\right)-\rho_{i} W_{\varepsilon}^{\prime \prime}\left(u_{j}-u_{i}\right)=2\left(\begin{array}{cccc}
1-\rho_{1} & -\rho_{1} & \cdots & -\rho_{1} \\
-\rho_{2} & 1-\rho_{2} & \cdots & -\rho_{2} \\
\vdots & \vdots & & \vdots \\
-\rho_{n} & -\rho_{n} & \cdots & 1-\rho_{n}
\end{array}\right) .
$$

restricted on the hyperspace $\left\{w_{i}: \sum_{i=1}^{n} w_{i}=0\right\}$ equals to the diagonal matrix $M=\operatorname{diag}(2)$ (use e.g. $w_{1}=-\sum_{i=2}^{n} w_{i}$ ). Altogether, we have constructed explicitly a class of stable (in the sense of Prop. 3.1) stationary states for any $n$ given masses $\rho_{i}$ satisfying $\frac{\rho_{i}+\rho_{i+1}}{2}>\varepsilon>\frac{\rho_{i}}{2}$ for $\varepsilon<\frac{1}{n}$.

We may now consider the weak limit of these stable stationary states. Observe, that by $(23)$ and $\sum_{i=1}^{n} \rho_{i}=1$ some straightforward calculations show

$$
u_{n}-u_{1}=1-\frac{\rho_{1}}{2}-\frac{\rho_{n}}{2}, \quad u_{n}+u_{1}=\frac{\rho_{1}}{2}-\frac{\rho_{n}}{2} .
$$

Thus, as $\rho_{i}<\frac{1}{n}$ we have $u_{1}=-\frac{1}{2}+\frac{\rho_{1}}{2} \rightarrow-\frac{1}{2}$ and $u_{n}=\frac{1}{2}-\frac{\rho_{n}}{2} \rightarrow \frac{1}{2}$ as $n \rightarrow \infty$. Then, for a given test function $\phi \in C_{c}(\mathbb{R})$ we calculate using (23) 


$$
\begin{aligned}
\int_{\mathbb{R}} \phi(x) d \bar{\rho}(x) & =\sum_{i=1}^{n} \phi\left(u_{i}\right) \rho_{i}=\sum_{i=1}^{n} \int_{u_{i}-\frac{\rho_{i}}{2}}^{u_{i}+\frac{\rho_{i}}{2}} \phi\left(u_{i}\right) d x \\
& =\int_{u_{1}-\frac{\rho_{1}}{2}}^{u_{n}+\frac{\rho_{n}}{2}} \sum_{i=1}^{n} \phi\left(u_{i}\right) \mathbb{1}_{\left[u_{i}-\frac{\rho_{i}}{2}, u_{i}+\frac{\rho_{i}}{2}\right]} d x \\
& \rightarrow \int_{-\frac{1}{2}}^{\frac{1}{2}} \phi(x) d x=\int_{\mathbb{R}} \mathbb{1}_{\left[-\frac{1}{2}, \frac{1}{2}\right]} \phi(x) d x,
\end{aligned}
$$

as $\rho_{i}<\frac{1}{n}$ and since $\phi$ is continuous.

The characteristic function $\rho=\mathbb{1}_{\left[-\frac{1}{2}, \frac{1}{2}\right]}$ is indeed the unique stationary state $\rho \in L^{\infty}(\mathbb{R})$ of the limiting potential $W(x)=x^{2}-|x|$. In fact, we have on the support of $\rho(x)$

$$
\begin{aligned}
0 & =\left(W^{\prime} * \rho\right)(x)=\int_{\mathbb{R}} 2(x-y) d \rho(y)-\int_{\mathbb{R}} \operatorname{sign}(x-y) d \rho(y) \\
& =2 x-\int_{-\infty}^{x} d \rho(y)+\int_{x}^{-\infty} d \rho(y) .
\end{aligned}
$$

Hence, taking the derivative in $x$, we obtain $0=2-2 \rho$ on the support of $\rho$. This yields the stationary state $\bar{\rho}=\mathbb{I}_{\left[-\frac{1}{2}, \frac{1}{2}\right]}$, which is moreover unique as one can check that any gap within the support of $\rho$ being stationary state of $W=x^{2}-|x|$ requires $\rho$ to have a Dirac mass at the edges of such a gap.

Example 4.1 (Non-uniqueness and numerical simulations). We remark that the above constructed stable stationary states of the regular potential $W_{\varepsilon}$ i) do not depend on the particular smoothing how $W_{\varepsilon} \rightarrow W$ (except that $W_{\varepsilon}^{\prime}(0)=0$ and $W_{\varepsilon}^{\prime \prime}(0)=\frac{1}{\varepsilon}$ ) and ii) are obviously non-unique since the masses $\rho_{i}$ can be chosen arbitrarily within the limits $\frac{\rho_{i}+\rho_{i+1}}{2}>\varepsilon>\frac{\rho_{i}}{2}$ for $\varepsilon<\frac{1}{n}$. Nevertheless, all these stationary state converge towards the unique stationary state $\rho=\mathbb{1}_{[-0.5,0.5]}$ of the singular repulsive potential $W=x^{2}-|x|$.

To illustrate the non-uniqueness of the stationary states of the potentials $W_{\varepsilon}(x)$ regard first Figure 3 for a softly smoothed modulus $|x|_{\varepsilon}$ with $\varepsilon=0.4$ : Four smoothed initial Diracs converge towards a two Dirac stationary state.

Secondly, for increasing local repulsion, i.e. $\varepsilon=0.18$, we observe nonuniqueness also in the number of Diracs: Observe how different initial data with three or four smoothed Dirac converge to stable three or four Diracs stationary state (see Figures 4 and 5).

Finally, Figure 6 shows convergence towards a multiple Dirac stationary state for strong local repulsion $\varepsilon=0.03$.

In the following we prove convergence of the stable steady-states for interaction potentials, which approximate the singular repulsive potential $W(x)=-|x|$, 

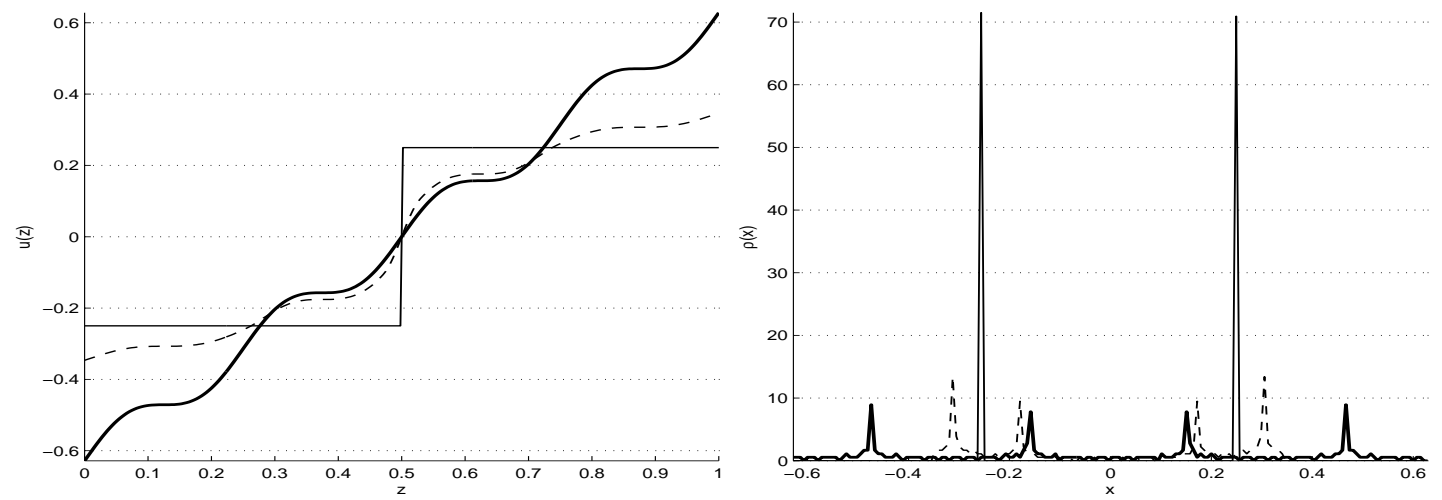

Figure 3. Convergence towards a stable symmetric two Dirac stationary state for the smoothed double-well potential $W(x)=$ $x^{2}-|x|_{\varepsilon}$ with $\varepsilon=0.4$. The left image plots $u(t, z)$ at time $t=0$ (bold line, initial data), $t=2$ (dashed line) and $t=25$ (stable stationary state, solid line). The right image plots the measures $\rho(t, x)$ at times $t=0$ (bold line), $t=2$ (dashed line) and $t=25$ (solid line).

where we define $W^{\prime}(0)=\operatorname{sign}(0)=0$. In particular, we shall consider stationary states confined by an external potential $V(x)$, i.e.

$$
\partial_{t} \rho(t, x)=\partial_{x}\left(\rho\left(W^{\prime} * \rho+V^{\prime}\right)\right),
$$

and the stationary states of an approximating, piecewise $C^{2}$ potential $W_{\varepsilon}(x)$ with $W_{\varepsilon}^{\prime}(0)=0,\left.W_{\varepsilon}^{\prime \prime}\right|_{(-\varepsilon, \varepsilon)}=-C_{\varepsilon}<0$ and $W_{\varepsilon}(x)=W(x)$ outside the interval $(-\varepsilon, \varepsilon)$

$$
\partial_{t} \rho_{\varepsilon}(t, x)=\partial_{x}\left(\rho_{\varepsilon}\left(W_{\varepsilon}^{\prime} * \rho_{\varepsilon}+V^{\prime}\right)\right) \text {, }
$$

The following proposition proves the weak convergence of the stationary states $\bar{\rho}_{\varepsilon} \rightarrow \bar{\rho}$ as $\varepsilon \rightarrow 0$.

Proposition 4.1. Let $V \in C^{2}(\mathbb{R})$ with $V^{\prime \prime} \geq \kappa>0$. Suppose that $\rho \in L^{\infty}(\mathbb{R})$ is a steady state of (24) and $\rho_{\varepsilon}$ a stable steady state of (25), stable in the sense that:

$$
0<\left(W_{\varepsilon} * \rho_{\varepsilon}+V\right)^{\prime \prime}(x) \quad \forall x \in \operatorname{supp}\left(\rho_{\varepsilon}\right)
$$

Then,

$$
\rho_{\varepsilon} \rightarrow \rho \quad \text { in } M^{1} \quad \text { as } \varepsilon \rightarrow 0 .
$$

Proof of Proposition 4.1. We divide the proof in four steps.

Step 1: The stationary state $\rho_{\varepsilon}$ cannot concentrate mass too much in the sense that

$$
\int_{x-\varepsilon}^{x+\varepsilon} \rho_{\varepsilon} \leq C_{1} \varepsilon
$$



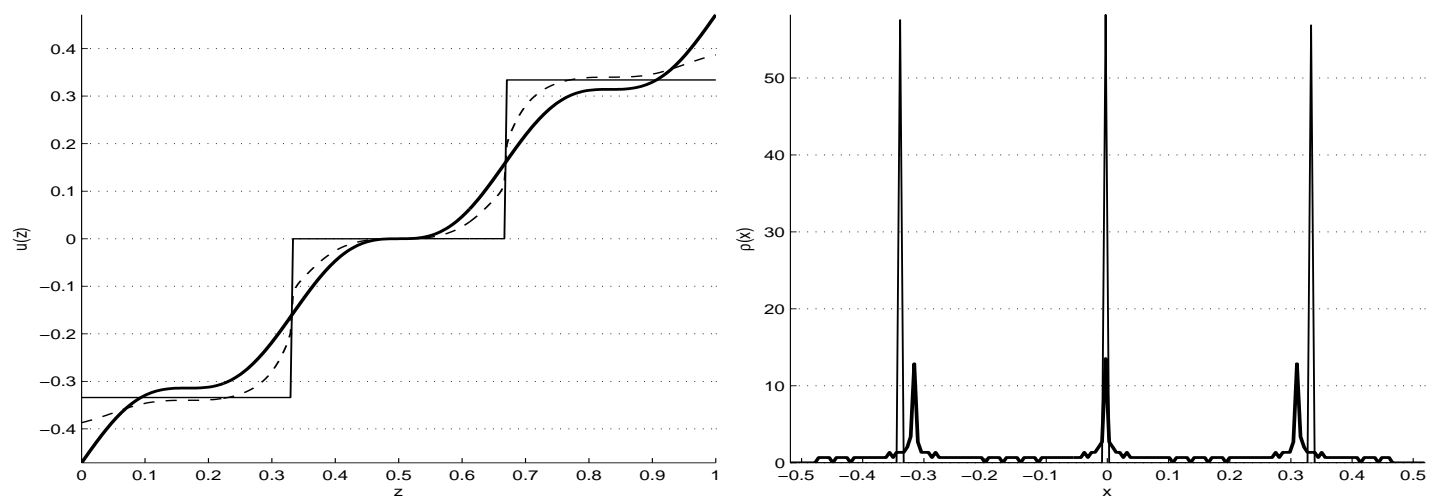

Figure 4. Convergence to a stable symmetric three Dirac stationary state for the smoothed double-well potential $W(x)=$ $x^{2}-|x|_{\varepsilon}$ with $\varepsilon=0.18$ subject initial data consisting of three smoothed Diracs. The left image plots $u(t, z)$ at time $t=0$ (bold line, initial data), $t=6$ (dashed line) and $t=80$ (stable stationary state, solid line). The right image plots the measures $\rho(t, x)$ at times $t=0$ (bold line) and $t=80$ (solid line).
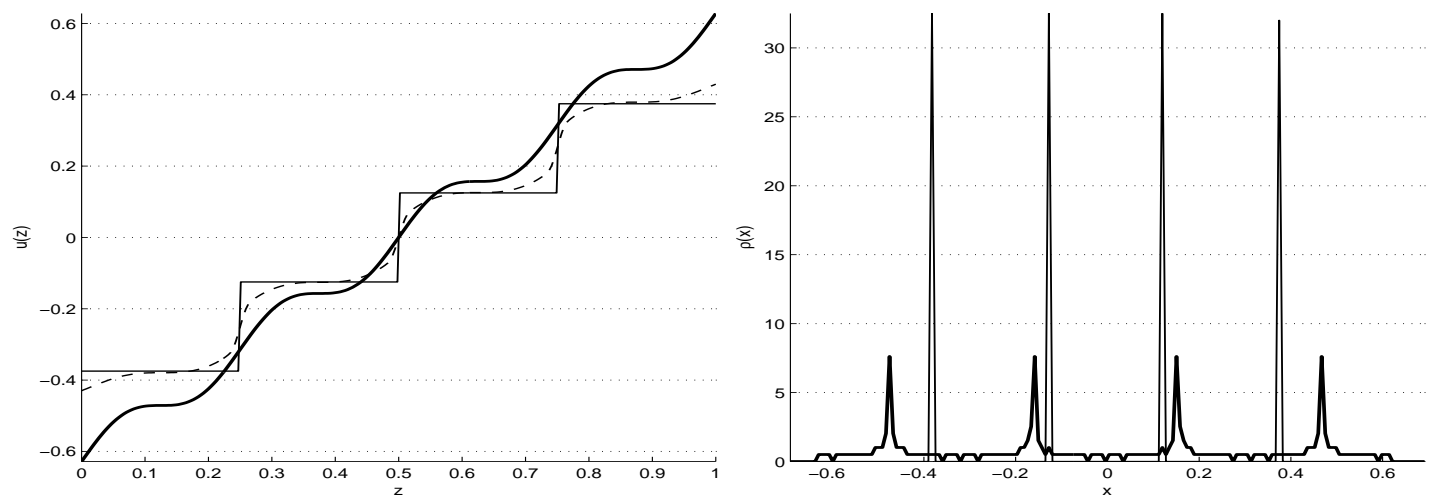

FiguRE 5. Convergence towards a stable symmetric four Dirac stationary state for the smoothed double-well potential $W(x)=$ $x^{2}-|x|_{\varepsilon}$ with $\varepsilon=0.18$ subject initial data consisting of four smoothed Diracs. The left image plots $u(t, z)$ at time $t=0$ (bold line, initial data), $t=2$ (dashed line) and $t=50$ (stable stationary state, solid line). The right image plots the measures $\rho(t, x)$ at times $t=0$ (bold line) and $t=50$ (solid line).

where $C_{1}=C_{1}\left(\left\|W^{\prime \prime}\right\|_{L^{\infty}(\mathbb{R} /\{0\})},\left\|V^{\prime \prime}\right\| L^{\infty}(\mathbb{R})\right)$. In fact, for $x \in \operatorname{supp}\left(\rho_{\varepsilon}\right)$ with $\rho_{\varepsilon}$ satisfying (26), we estimate

$$
0 \leq\left(W_{\varepsilon} * \rho_{\varepsilon}+V\right)^{\prime \prime}(x) \leq-\frac{C_{1}}{\varepsilon} \int_{x-\varepsilon}^{x+\varepsilon} \rho_{\varepsilon}+\left\|W^{\prime \prime}\right\|_{L^{\infty}\left([-\varepsilon, \varepsilon]^{c}\right)}\left\|\rho_{\varepsilon}\right\|_{M^{1}}+\left\|V^{\prime \prime}\right\|_{\infty} .
$$



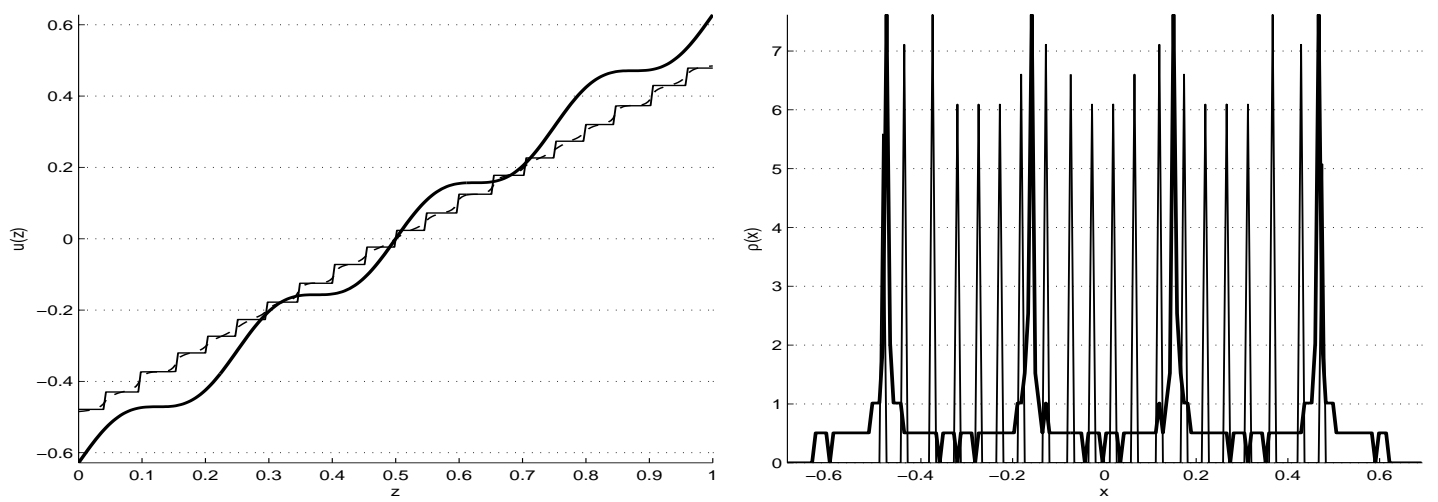

Figure 6. Convergence towards a stable symmetric multiple Dirac stationary state for the smoothed double-well potential $W(x)=x^{2}-|x|_{\varepsilon}$ with $\varepsilon=0.03$ subject initial data consisting of four smoother Diracs. The left image plots $u(t, z)$ at time $t=0$ (bold line, initial data), $t=4$ (dashed line) and $t=80$ (stable stationary state, solid line). The right image plots the measures $\rho(t, x)$ at times $t=0$ (bold line) and $t=80$ (solid line).

Step 2: The stationary state $\rho_{\varepsilon}$ cannot spread out too much in the sense that if $x \in \operatorname{conv}\left(\operatorname{supp}\left(\rho_{\varepsilon}\right)\right)$, then there exist $\tilde{x} \in \operatorname{supp}\left(\rho_{\varepsilon}\right)$ such that $|x-\tilde{x}| \leq C_{2} \varepsilon$, where $C_{2}=C_{2}\left(C_{1},\left\|W^{\prime}\right\|_{L^{\infty}(\mathbb{R})}, \kappa\right)$. Indeed, suppose a gap within the $\operatorname{supp}\left(\rho_{\varepsilon}\right)$ between the points $x_{1}, x_{2} \in \operatorname{supp}\left(\rho_{\varepsilon}\right)$, i.e. the interval $\left(x_{1}, x_{2}\right) \notin \operatorname{supp}\left(\rho_{\varepsilon}\right)$. Then,

$$
\begin{aligned}
0= & \left(W_{\varepsilon} * \rho_{\varepsilon}+V\right)^{\prime}\left(x_{2}\right)-\left(W_{\varepsilon} * \rho_{\varepsilon}+V\right)^{\prime}\left(x_{1}\right) \\
= & \int_{\mathbb{R}}\left(W_{\varepsilon}^{\prime}\left(x_{2}-y\right)-W_{\varepsilon}^{\prime}\left(x_{1}-y\right)\right) d \rho_{\varepsilon}(y)+V^{\prime}\left(x_{2}\right)-V^{\prime}\left(x_{1}\right) \\
= & \int_{x_{1}-\varepsilon}^{x_{1}}\left(W_{\varepsilon}^{\prime}\left(x_{2}-y\right)-W_{\varepsilon}^{\prime}\left(x_{1}-y\right)\right) d \rho_{\varepsilon}(y) \\
& +\int_{x_{2}}^{x_{2}+\varepsilon}\left(W_{\varepsilon}^{\prime}\left(x_{2}-y\right)+W_{\varepsilon}^{\prime}\left(x_{1}-y\right)\right) d \rho_{\varepsilon}(y)+V^{\prime \prime}\left(x_{\theta}\right)\left(x_{2}-x_{1}\right),
\end{aligned}
$$

with $x_{\theta} \in\left(x_{1}, x_{2}\right)$. Thus, thank to $(27)$ in step 1 , we estimate

$$
0 \geq-2\left\|W_{\varepsilon}^{\prime}\right\|_{L^{\infty}(\mathbb{R})} C_{1} \varepsilon+\kappa\left(x_{2}-x_{1}\right)
$$

Step 3: $\operatorname{supp}\left(\rho_{\varepsilon}\right) \subset \operatorname{supp}(\rho)$ and almost all the mass of $\rho$ is in $\operatorname{conv}\left(\operatorname{supp}\left(\rho_{\varepsilon}\right)\right)$.

First, since $V$ is convex, $\operatorname{supp}(\rho)$ is essentially convex, in the sense that $\operatorname{supp}(\rho)$ is dense in $\operatorname{conv}(\operatorname{supp}(\rho))$. As $\rho \in L^{\infty}$ by Proposition 2.1, this follows similar to 
step 2 for an interval $\left(x_{1}, x_{2}\right) \nsubseteq \operatorname{supp}(\rho)$ from

$$
\begin{aligned}
0 & =\left(W^{\prime} * \rho\right)\left(x_{2}\right)-\left(W^{\prime} * \rho\right)\left(x_{1}\right)+V^{\prime}\left(x_{2}\right)-V^{\prime}\left(x_{1}\right) \\
& =-2 \int_{x_{1}}^{x_{2}} \rho d x+V^{\prime \prime}(\theta)\left(x_{2}-x_{1}\right)=V^{\prime \prime}(\theta)\left(x_{2}-x_{1}\right) .
\end{aligned}
$$

Next, we consider the left points $x:=\min \{y \in \mathbb{R} ; y \in \operatorname{supp}(\rho)\}$, and $x_{\varepsilon}:=$ $\min \left\{y \in \mathbb{R} ; y \in \operatorname{supp}\left(\rho_{\varepsilon}\right)\right\}$. Thus,

$$
W^{\prime} * \rho(x)=\int_{\operatorname{supp}(\rho) /\{x\}} \rho d x=1, \quad W^{\prime} * \rho_{\varepsilon}\left(x_{\varepsilon}\right)=\int_{\operatorname{supp}\left(\rho_{\varepsilon}\right) /\left\{x_{\varepsilon}\right\}} \rho_{\varepsilon} d x \leq 1,
$$

and, as $\rho$ and $\rho_{\varepsilon}$ are both steady states, we have

$$
V^{\prime}(x)=W^{\prime} * \rho(x) \leq W^{\prime} * \rho_{\varepsilon}\left(x_{\varepsilon}\right)=V^{\prime}\left(x_{\varepsilon}\right)
$$

An analog estimates holds for the right points $y:=\max \{y \in \mathbb{R} ; y \in \operatorname{supp}(\rho)\}$, and $y_{\varepsilon}:=\max \left\{y \in \mathbb{R} ; y \in \operatorname{supp}\left(\rho_{\varepsilon}\right)\right\}$. Thus, since $V$ is convex, we conclude

$$
\operatorname{supp}\left(\rho_{\varepsilon}\right) \subset \operatorname{supp}(\rho) .
$$

Moreover, for $x_{\varepsilon} \in \operatorname{supp}\left(\rho_{\varepsilon}\right) \subset \operatorname{supp}(\rho)$ it follows also from (28) that $\left.W^{\prime} * \rho\right)\left(x_{\varepsilon}\right)+$ $V^{\prime}\left(x_{\varepsilon}\right)=0$. Thus,

$$
\begin{aligned}
0 & =\left(\left(W^{\prime} * \rho\right)\left(x_{\varepsilon}\right)+V^{\prime}\left(x_{\varepsilon}\right)\right)-\left(\left(W_{\varepsilon}^{\prime} * \rho_{\varepsilon}\right)\left(x_{\varepsilon}\right)+V^{\prime}\left(x_{\varepsilon}\right)\right) \\
& =\left(W^{\prime} *\left(\rho-\rho_{\varepsilon}\right)\right)\left(x_{\varepsilon}\right)+\left(\left(W^{\prime}-W_{\varepsilon}^{\prime}\right) * \rho_{\varepsilon}\right)\left(x_{\varepsilon}\right) \\
& =-2 \int_{-\infty}^{x_{\varepsilon}}\left(\rho-\rho_{\varepsilon}\right) d x+O(\varepsilon) .
\end{aligned}
$$

That is $\int_{-\infty}^{x_{\varepsilon}} \rho d x=O(\varepsilon)$. Repeating this argument on the right side of the support, we get

$$
\int_{\operatorname{supp}(\rho) \backslash \operatorname{supp}\left(\rho_{\varepsilon}\right)} \rho d x=O(\varepsilon)
$$

Step 4: Weak convergence of $\rho_{\varepsilon} \rightarrow \rho$.

It is sufficient to show that for any $x \in \mathbb{R}$,

$$
\int_{-\infty}^{x}\left(\rho-\rho_{\varepsilon}\right) d x \rightarrow 0
$$

If $x \notin \operatorname{conv}(\operatorname{supp}(\rho))$, this follows obviously from $\operatorname{supp}\left(\rho_{\varepsilon}\right) \subset \operatorname{supp}(\rho)$. If $x \in$ $\operatorname{conv}(\operatorname{supp}(\rho))$, then we have either $x \notin \operatorname{conv}\left(\operatorname{supp}\left(\rho_{\varepsilon}\right)\right)$, and therefore by step 3 ,

$$
\int_{-\infty}^{x}\left(\rho-\rho_{\varepsilon}\right) d x \leq \int_{\operatorname{supp}(\rho) \backslash \operatorname{supp}\left(\rho_{\varepsilon}\right)} \rho d x=O(\varepsilon),
$$


or we have $x \in \operatorname{conv}\left(\operatorname{supp}\left(\rho_{\varepsilon}\right)\right)$, and thus, by step 2 that there exist $\tilde{x} \in \operatorname{supp}\left(\rho_{\varepsilon}\right)$ such that $\left|x-x_{\varepsilon}\right| \leq C \varepsilon$, and

$$
\begin{aligned}
\int_{-\infty}^{x} \rho_{\varepsilon} d x & =\int_{-\infty}^{\tilde{x}} \rho_{\varepsilon} d x+O(\varepsilon)=\frac{1}{2}\left(1-W^{\prime} * \rho_{\varepsilon}\right)+O(\varepsilon) \\
& =\frac{1}{2}\left(1-W_{\varepsilon}^{\prime} * \rho_{\varepsilon}\right)+O(\varepsilon)=\int_{-\infty}^{\tilde{x}} \rho d x+O(\varepsilon) \\
& =\int_{-\infty}^{x} \rho d x+O(\varepsilon)
\end{aligned}
$$

This ends to proof of Proposition 4.1.

\section{ACKNOWLEDGEMENTS}

Both authors would like to thank very much Prof. Christian Schmeiser for initiating the research done in this article and Dr. Marco Di Francesco for many valuable discussions. KF has been supported by Award No. KUK-I1-007-43 of Peter A. Markowich, made by King Abdullah University of Science and Technology (KAUST) and by the bilateral Austria-France project (Austria: FR 05/2007 France: Amadeus 13785 UA). GR has partially been supported by the DEASE program affiliated at the WPI, Wolfgang Pauli Institute, University of Vienna.

\section{REFERENCES}

[Bee] P. R. Beesak, Gronwall inequalities. Carleton Math. Lecture Notes No.11 (1975).

[BCP] D. Benedetto, E. Caglioti, M. Pulvirenti, A kinetic equation for granular media, RAIRO Modél. Math. Anal. Numér., 31 (1997) 615-641.

[BKR] E. Ben-Naim, P.L. Krapivsky, S. Redner, Bifurcations and Patterns in Compromise Processes, Physica D 183 (2003) 190-204.

[BB] A.L. Bertozzi, J. Brandman, Finite-time blow-up of $L^{\infty}$-weak solutions of an aggregation equation, to appear in Comm. Math. Sci.

[BCL] A. Bertozzi, J.A. Carrillo, T. Laurent, Blowup in multidimensional aggregation equations with mildly singular interaction kernels, Nonlinearity 22 (2009), 683-710.

[BL] A. Bertozzi, T. Laurent, Finite-time Blow-up of Solutions of an Aggregation Equation in $R^{n}$, Comm. Math. Phys. 274(3) (2007) 717-735.

[BCC] A. Blanchet, V. Calvez, J.A. Carrillo, Convergence of the mass-transport steepest descent scheme for the sub-critical Patlak-Keller-Segel model, SIAM J. Numer. Anal. 46 (2008) 691-721.

[BDP] A. Blanchet, J. Dolbeault, B. Perthame, Two-dimensional Keller-Segel model: optimal critical mass and qualitative properties of the solutions, Electron. J. Differential Equations 44 (2006).

[BCM00] S. Boi, V. Capasso, D. Morale Modeling the aggregative behavior of ants of the species Polyergus rufescens Spatial heterogeneity in ecological models (Alcala de Henares 1998), Nonlinear Anal. Real World Appl. 1 (2000) 163-176.

[BCM07] M. Burger, V. Capasso, D. Morale On an aggregation model with long and short range interactions Nonlinear Analysis. Real World Applications 8 (2007) 939-958.

[BDiF] M. Burger, M. Di Francesco, Large time behavior of nonlocal aggregation models with non-linear diffusion, Netw. Heterog. Media 3 (2008) 749 - 785. 
[CDFLS] J.A. Carrillo, M. Di Francesco, A. Figalli, T. Laurent, D. Slepčev, Global-in-time weak measure solutions, finite-time aggregation and confinement for nolocal interaction equations, preprint UAB 17 (2009), submitted.

[CMV] J.A. Carrillo, R.J. McCann, C. Villani, Kinetic equilibration rates for granular media and related equations: entropy dissipation and mass transportation estimates, Rev. Mat. Iberoamericana 19 (2003), 971-1018.

[CR] J. A. Carrillo, J. Rosado, Uniqueness of bounded solutions to sggregation equations by optimal transport methods preprint.

[CT] J.A. Carrillo, G. Toscani, Wasserstein metric and large-time asymptotics of nonlinear diffusion equations. New trends in mathematical physics, 234-244, World Sci. Publ., Hackensack, NJ, 2004.

[CH] Y.-L. Chuang, Y. R. Huang, M. R. D’Orsogna, A. L. Bertozzi, Multi-vehicle flocking: scalability of cooperative control algorithms using pairwise potentials IEEE International Conference on Robotics and Automation (2007) 2292-2299.

[KPSV] K. Kang, B. Perthame, A. Stevens, J.J.L. Velázquez, An integro-differential equation model for alignment and orientational aggregation, J. Differential Equations 246(4) (2009) 1387-1421.

[Lau] T. Laurent, Local and Global Existence for an Aggregation Equation, Comm. Partial Differential Equations, 32 (2007) 1941-1964.

[LT] H. Li, G. Toscani, Long-time asymptotics of kinetic models of granular flows. Arch. Ration. Mech. Anal. 172(3) (2004) 407-428.

[MEK] A. Mogilner, L. Edelstein-Keshet, A non-local model for a swarm, J. Math. Bio. 38 (1999) 534-570.

[MCO] D. Morale, V. Capasso, K. Oelschlager An interacting particle system modelling aggregation behavior: from individuals to populations J. Math. Biol. 50 (2005) 49-66.

[Pat] C.S. Patlak, Random walk with persistence and external bias, Bull. Math. Biophys. 15 (1953) 311-338.

[PSV] I. Primi, A. Stevens, J. J.L. Velazquez, Mass-Selection in Alignment Models with Non-Deterministic Effects, Comm. Partial Differential Equations 34(5) (2009).

[Rao1] G. Raoul, Local stability of evolutionary attractors for continuous structured populations, preprint CMLA-ENS Cachan 1 (2009), submitted.

[Rao2] G. Raoul, Non-local interaction equations : Stationary states and stability analysis, preprint CMLA-ENS Cachan 25 (2009), submitted.

[Rao3] G. Raoul, Orbital stability of some steady-states for an aggregation equation, work in progress.

[TB] C.M. Topaz, A.L. Bertozzi, Swarming patterns in a two-dimensional kinematic model for biological groups SIAM J. Appl. Math. 65 (2004) 152-174.

[TBL] C.M. Topaz, A.L. Bertozzi, M.A. Lewis, A nonlocal continuum model for biological aggregation, Bull. Math. Biol. 68(7) (2006) 1601-1623.

[The] F. Theil, A proof of crystallization in two dimensions, Comm. Math. Phys. 262(1) (2006) 209-236.

[Tos] G. Toscani, One-dimensional kinetic models of granular ows RAIRO Modél. Math. Anal. Numér. 34 (2000) 1277-1291.

KLEMENS FELLnER

DAMTP, CMS, University OF CAMBridge

Wilberforce RoAD

Cambridge CB3 0WA, United Kingdom

E-mail address, K.FELLNER@DAMTP.CAM.AC.UK: 
On LEAVE From: FACUlty of Mathematics

UNIVERSITY OF VIENNA

Nordbergstr. 15, 1090 Wien, Austria

E-mail address: Klemens.Fellner@univie.ac.at

Gä̈L RAOUL

CMLA, ENS Cachan, CNRS, PRES UniverSud

61 Av. Du PdT. Wilson

94235 CaChan Cedex, France

E-mail address: gael.raoul@cmla.ens-cachan.fr 